\title{
Krise des Weltkriegs und Transformation eines Wissenschaftsfeldes: Ethnographie und Anthropologie in Österreich-Ungarn und Deutschland bis 1914/18 und danach
}

Der vorliegende Beitrag behandelt die Veränderungen, welche deutschsprachige Anthropologie und Ethnographie im Zuge des Ersten Weltkriegs durchliefen. Dabei kann davon ausgegangen werden, dass der Erste Weltkrieg für die K.u.K. Monarchie und das deutsche Kaiserreich in seinem Gesamtverlauf einer krisenhaften Zuspitzung gleichkam, die in den militärischen, gesellschaftlichen und politischen Zusammenbruch dieser beiden Systeme mündete. Da das Generalthema dieses Bandes die Wechselwirkungen zwischen Krise und Transformation aufwirft, wird der politisch-militärische Gesamtverlauf des Ersten Weltkriegs in Deutschland und ÖsterreichUngarn hier als Grundlage eben einer derartigen „Krise“ befragt, in deren Rahmen sich die „Transformation“ des damaligen wissenschaftlichen Fächerbündels aus Ethnographie und Anthropologie vollzog. ${ }^{1}$

Aus der retrospektiven Langzeit-Perspektive des frühen 21. Jh. betrachtet, wirkte der Erste Weltkrieg für die Entwicklung von Anthropologie und Ethnographie als großer Katalysator, durch den wissenschaftsinterne Entwicklungen in Deutschland und Österreich-Ungarn beschleunigt, verschärft und in bestimmte Richtungen vorangetrieben wurden. Vorab kann festgehalten werden, dass der zeitgenössische Forschungsstand keinen Zweifel dahingehend zulässt, $o b$ die Krise des Ersten Weltkriegs überhaupt eine Transformation von Ethnographie und Anthropologie bewirkt habe. ${ }^{2}$ Offen sind eher zwei andere Fragen: Erstens, welcher Art waren diese wissenschaftsinternen Transformationen, und welches Gewicht kam dabei dem Moment von „Innovation“ zu? Zweitens, welche faktische Rolle spielten die wissenschaftlichen Beiträge aus deutschsprachiger Ethnographie und Anthropologie im Rahmen dieser wissenschaftlichen Transformationen für die letztlich erfolglosen Kriegsanstrengungen?

Diesen beiden Hauptfragen geht der vorliegenden $\mathrm{Text}^{3}$ in drei Abschnitten und einer Zusammenfassung nach. Dabei wird der Hauptakzent auf die Herausbildung langfristiger Entwicklungstendenzen gelegt, während Einzelfaktoren nur zusammengefasst werden. Der erste Abschnitt legt wesentliche Bestimmungen und Ausrichtungen von Ethnographie und Anthropologie in Deutschland und Österreich-Ungarn vor dem Ausbruch des Ersten Weltkriegs vergleichend dar. Der zweite Abschnitt diskutiert das einzigartige und

\footnotetext{
${ }^{1}$ Arnold Suppan ist der Autor dankbar verbunden für die Initiative zu diesem Band und zum vorangegangen Symposium sowie für die Einladung zu Beidem. Darüber hinaus dankt der Autor für ihre hilfreichen Kommentare Dr. Britta Lange (HU Berlin), mit der er 2008-2010 im Rahmen ihrer Förderung als Lise Meithner-Stipendiatin des Österreichischen Wissenschaftsfonds FWF am Institut für Sozialanthropologie der Österreichischen Akademie der Wissenschaften in den hier diskutierten Fragen zusammenarbeitete. Diese Zusammenarbeit nahm 2006 auf einer vom Ludwig Uhland Institut für Empirische Kulturwissenschaften der Universität Tübingen veranstalteten und aus Mitteln eines SFB der Deutschen Forschungsgemeinschaft finanzierten Konferenz ihren Ausgang. Der vorliegende Text fasst in wesentlichen Punkten Ergebnisse dieses mehrjährigen Arbeitsprozesses zusammen, und kann daher stets nur einen groben Überblick vermitteln. Für alle detaillierten historischen und wissenschaftsgeschichtlichen Quellenangaben muss auf die Anmerkungsapparate der hier ausschließlich zitierten Sekundärliteratur verwiesen werden. Verena Loidl (Institut für Sozialanthropologie der ÖAW) dankt der Autor für editorische Assistenz bei der Erstellung des vorliegenden Beitrags.

2 Siehe dazu die Beiträge in Barth, Fredrik, Gingrich, Andre, Parkin, Robert, Silvermann, Sydel: One Discipline, Four Ways: British, German, French, and American Anthropology, Chicago: Chicago University Press 2005; sowie in KukLICK, Henrika (Hg.): A New History of Anthropology, Oxford: Blackwell 2008.

${ }^{3}$ Für eine ausführlichere englischsprachige Version des vorliegenden Textes siehe im Tübinger Tagungsband: GiNGRICH, Andre: After the Great War: National Re-Configurations of Anthropology in Late Colonial Times, in: Johler, Reinhard, Marchetti, Christian, ScheER, Monique (eds.): Doing Anthropology in Wartime and War Zones, Bielefeld: transcript 2010, 355-379, und Lange, Britta: AfterMath: Anthropological Data from Prisoner-of-War Camps, in Johler et al.: Anthropology in Wartime 2010, 311-335; ferner Gingrich, Andre und LANGE, Britta: Gefangene Stimmen, Internierte Körper: Rudolf Pöch, die Wünsdorf-Reise 1917 und die Frage der Geschichte der Völkerkunde, in: Ballhausen, Thomas, Matiasek, Katarina, Teschler-Nicola, Maria (Hg.): Archivhorizonte: Wissenslandschaften und Perspektivgrenzen im multimedialen Nachlass des Anthropologen und Forschungsreisenden Rudolf Pöch, Wien: Löcker i.Dr.
} 
besondere Engagement dieser Teile von Ethnographie und Anthropologie während des Großen Krieges. Der dritte Abschnitt demonstriert schließlich anhand der Situation nach ungefähr einem Jahrzehnt, also zu Ende der 1920er-Jahre, auf welche institutionelle und personelle Gesamtentwicklung die Transformationen des Ersten Weltkrieges wissenschaftsintern allmählich für Ethnographie und Anthropologie im deutschsprachigen Raum hinausliefen. Aus diesen drei Abschnitten wird am Ende ein zusammenfassendes Fazit gezogen, das die hier formulierten beiden Hauptfragen nochmals anspricht und beantwortet.

\section{IMPERIALER LIBERALISMUS UND INTERDISZIPLINARITÄT: ETHNOGRAPHIE UND ANTHROPOLOGIE IM DEUTSCHSPRACHIGEN RAUM VOR 1914}

Wer heute das Naturhistorische Museum an der Wiener Ringstraße besucht, ist eingeladen, auch dessen eindrucksvollen, zentralen Kuppelraum zu betreten und den Blick nach oben zu richten. Kreisförmig sind dort rund um die eigentliche Kuppelbasis in goldenen Lettern die Namen jener großen Hauptdisziplinen eingetragen, welche bei der Errichtung des 1889 eröffneten Prachtbaus als wesentliche Grundlage der Naturgeschichte angesehen wurden. Als zwei dieser wenigen Hauptfächer prangen dort die Bezeichnungen „Ethnographie“ und „Anthropologie“.

Tatsächlich hatte das Wissensfeld dieser beiden Disziplinen einen seiner wesentlichen modernen Ausgangspunkte im deutschsprachigen Raum, und dort wiederum lagen deren primäre institutionelle Hauptstützen lange Zeit in den großen städtischen Museen. Das Konzept von „Ethnographie“ war im letzten Viertel des 18. Jh. vom aufgeklärten Göttinger Gelehrten August Ludwig von Schlözer als Analogie zu „Geographie“ entwickelt worden, um jenen empirischen Gegebenheiten Rechnung zu tragen, die in zunehmendem Maße als Folge europäischer Entdeckungsreisen auf allen Kontinenten das Wissen um die Sitten und Gebräuche der Menschheit bereicherten. Parallel dazu hatte ebenfalls in Göttingen Johann Friedrich Blumenbach begonnen, die noch spärlichen Einsichten in die physische Vielfalt der Menschen zum Fach Anthropologie aus zu bearbeiten. ${ }^{4}$ Dass es enge Zusammenhänge zwischen der biologischen und kulturellen Seite des Menschseins geben müsse, war in beiden Fachbereichen unbestritten. Wie diese zu beschreiben und zu erklären seien, blieb über lange Perioden Thema erbitterter wissenschaftlicher und immer auch weltanschaulicher Auseinandersetzungen.

Die Herder'sche Idee einer hierarchischen Unterscheidung in „Naturvölker“ und „Kulturvölker“ führte erst in weiterer Folge und sekundär dazu, dass „Ethnographie“ im Verlauf des 19. Jh. immer häufiger in die zwei entsprechenden Subdisziplinen „Völkerkunde“ (als Studium der „Naturvölker“) und „Volkskunde“ (als Erforschung der „Kulturvölker“, und dabei besonders der eigenen Kultur) untergliedert wurde. ${ }^{5}$ Diese Unterscheidung wurde mit größerem Engagement in Deutschland forciert, während man ihr aus guten Gründen im habsburgischen Vielvölkerstaat - ähnlich übrigens wie im zaristischen Russland, aber ebenso in der multiethnischen Schweiz - weitaus skeptischer gegenüberstand. In Österreich-Ungarn wurde also eigentlich bis 1918 „Ethnographie“ über die Sitten und Gebräuche sowohl „eigener“ wie ,anderer“ Kulturen betrieben auch unter dem imperialen Primat der erwünschten Kohärenz im Vielvölkerstaat. ${ }^{6}$ In der Praxis wirkten dabei zweifellos magyarische und deutschösterreichische Hegemonien, wobei aber deren allzu offizielle und explizite Zelebrierung eher vermieden wurde. Dem multiethnischen Territorialprinzip, das in Österreich-Ungarn eine liberalere und aus heutiger Sicht durchaus modernere Auffassung von „Ethnographie“ bevorzugte, wie sie etwa im „Kronprinzenwerk“ zum Ausdruck kam, ${ }^{7}$ stand in dem ab 1869/71 errichteten deutschen Kaiser- und

\footnotetext{
${ }^{4}$ Gingrich in Barth et al.: One Discipline, Four Ways 2005, 74; Vermeulen, Han: Origins and Institutionalization of Ethnography and Ethnology in Europe and the USA, 1771-1845, in: Vermeulen, Han and Roldan, Arturo Alvarez (eds.): Fieldwork and Footnotes: Studies in the History of European Anthropology, London: Routledge 1995, 39-59.

${ }^{5}$ Zамміто, John H.: Kant, Herder and the Birth of Anthropology, Chicago: University of Chicago Press 2002.

${ }^{6}$ Stachel, Peter: Die Harmonisierung national-politischer Gegensätze und die Anfänge der Ethnographie in Österreich, in: Aснам, Karl (Hg.): Geschichte der österreichischen Humanwissenschaften, Bd. 4: Geschichte und fremde Kulturen. Wien: Passagen 2002, 323-368.

${ }^{7}$ BENDIX, Regina: Ethnology, Cultural Reification, and the Dynamics of Difference in the Kronprinzenwerk, in: WingrieLd, Nancy M. (ed.): Creating the Other: Ethnic Conflict and Nationalism in the Habsburg Empire. New York - London: Berghahn 2003, 149-165.
} 
Kolonialreich eine immer striktere hierarchische Trennung von „Volks-“ und „Völkerkunde“ gegenüber. Sie entsprach im wesentlichen der Bismarck'schen Ausrichtung auf einen deutschen Nationalstaat im Inneren, der nach außen hin zugleich als relativer Spätankömmling besonders energische Ambitionen auf Kolonien in Übersee umsetzte, insbesondere in Afrika und Melanesien. Allerdings setzte sich auch in Deutschland bis 1918 eine eigene „Volkskunde“ - trotz ihrer stärkeren Ausdifferenzierung in eigenen Zeitschriften und ersten Museen - noch nicht als selbstständiges universitäres Fach durch.

Bis zur Wende vom 19. zum 20. Jh. verlief die Zusammenarbeit zwischen der sich mehr oder minder in Volksund Völkerkunde ausdifferenzierenden Ethnographie und der (physischen oder biologischen) Anthropologie insgesamt relativ ausgewogen. Das lag sowohl am noch vorherrschenden Zeitgeist der Friedensperiode als auch an wissenschaftsinternen Faktoren, von denen dreien ein besonderes Gewicht zukam. Erstens arbeitete die physische Anthropologie noch primär mit metrischen und phänomenologischen Methoden, was ihren Aussagen eher induktiven und explorativen Charakter verlieh und ihr ein geringeres Ausmaß an Beanspruchung von kausaler Reichweite und an damit einher gehendem Sendungsbewusstsein ermöglichte. Zweitens waren die meisten Fachvertreter noch universitär ausgebildete Ärzte oder Historiker. Diese fühlten sich in einem durchaus beachtlichen Ausmaß den eher humanistischen Traditionen der Wissenschaftsgeschichte verpflichtet und betrieben sehr häufig ihre eigene Spezialisierung in ethnographischen ebenso wie in physisch-anthropologischen Bereichen. ${ }^{8}$ Drittens schließlich boten die großen Museen in Berlin, Wien, Basel oder Leipzig stets beiden Fachbereichen eine gemeinsame Heimat - wie es eben auch in den goldenen Inschriften unter der Kuppel des Wiener NHM zum Ausdruck gebracht wurde. Diese gemeinsame institutionelle Heimstätte förderte unter den damaligen Bedingungen in der Regel eher die Kooperation als die Rivalität. Herausragendes und bekanntestes Beispiel dafür war das Berliner Museum für Völkerkunde. ${ }^{9}$

Länger als ein Vierteljahrhundert führten die beiden promovierten Ärzte Adolf Bastian (Völkerkunde) und Rudolph Virchow (Anthropologie) das Berliner Museum für Völkerkunde als Direktoren und deutsche Gründergestalten ihrer Fächer. ${ }^{10}$ Aufbauend auf den positiven Erfahrungen mit den älteren und durch bürgerlich-städtische Interessen als öffentliche Bildungsanstalten getragenen Museen in Basel und Leipzig ${ }^{11}$ machten Virchow und Bastian das Berliner Museum zum größten Fachmuseum der Welt, das es bis weit in die 1930er-Jahre bleiben sollte. Umfangreiche Erhebungen seitens der Anthropologie im Inland, wie vor allem Virchows „Schulstatistik“, ${ }^{12}$ und seitens der Völkerkunde im Ausland, wie insbesondere Bastians rastlose Sammlungsreisen auf allen Kontinenten ${ }^{13}$ legten den Grundstein zum Ausbau des Berliner Museums hin zu einer Schatzkammer von geradezu enzyklopädischem Charakter, in der physische Anthropologie und Ethnographie koexistierten - manchmal eher nebeneinander, oft aber auch miteinander. Neben der empirischen und dokumentarischen Seite bildete die institutionelle die zweite wichtige Komponente dieser Phase, geprägt vor allem auch durch die interdisziplinären wissenschaftlichen Vereine. Dies waren in erster Linie die Berliner Gesellschaft für Anthropologie, Ethnologie und Urgeschichte sowie die Anthropologische Gesellschaft zu Wien. ${ }^{14}$ In der Hauptstadt der Donaumonarchie hatte die offizielle Skepsis gegenüber allzu großer Abtrennung von Volks- und Völkerkunde 1895 zur bloß privaten Gründung des Museums für Volkskunde geführt, während seit 1876 eine stetig wachsende ,anthropologisch-ethnographische Abteilung“" vorerst weiter (bis 1928) im Naturhistorischen Museum belassen wurde. Später führende heimische Exponenten des Faches zogen nach

\footnotetext{
${ }^{8}$ Massin, Benoît: From Virchow to Fischer: Physical anthropology and „modern race theories“ in Wilhelmine Germany, in: STOCKING, George W. Jr. (ed.): Volksgeist as Method and Ethic: Essays on Boasian ethnography and the German anthropological tradition, History of Anthropology vol. 8, Madison: University of Wisconsin Press 1996, 79-154.

9 Penny, Glenn H. and Bunzl, Matti (eds.): Worldly Provincialism: German anthropology in the age of empire. Ann Arbor: University of Michigan Press 2003.

${ }^{10}$ Koepping, Klaus Peter: Adolf Bastian and the Psychic Unity of Mankind: The Foundations of Anthropology in Nineteenth Century Germany. St. Lucia: University of Queensland Press 1983.

${ }^{11}$ Penny, Glenn H. and Bunzl, Matti: Worldly Provincialism, 11.

${ }_{12}$ Zimmermann, Andrew: Anthropology and Antihumanism in Imperial Germany. Chicago - London: University of Chicago Press 2001.

${ }_{13}$ Koepping, Klaus-Peter, Bastian, 1983.

${ }_{14}$ Pusman, Karl: Die "Wissenschaften vom Menschen" auf Wiener Boden (1870-1959): Die Anthropologische Gesellschaft in Wien und die anthropologischen Disziplinen im Fokus von Wissenschaftsgeschichte, Wissenschafts- und Verdrängungspolitik, Münster: LiT 2008.
} 
Berlin, um bei Virchow und Bastian ihre Ausbildung zu erhalten: Felix von Luschan aus Niederösterreich wurde in Berlin nach 1900 der zentrale Nachfolger; der Wiener Richard Thurnwald wurde vor 1914 Student und junger Mitarbeiter in Berlin (er sollte ab den 1920er Jahren von Berlin aus zum wohl weltweit bekanntesten deutschsprachigen Völkerkundler der ersten Hälfte des 20. Jh. werden); Rudolf Pöch schließlich hatte bei von Luschan in Berlin studiert und erhielt 1912 an der Universität Wien die erste Professur für Ethnographie und Anthropologie zugesprochen: In der Wiener Anthropologischen Gesellschaft wie in der Kaiserlichen Akademie prägte er das Fach maßgeblich mit. ${ }^{15}$

International waren die deutschsprachige Ethnographie und Anthropologie der Jahrzehnte bis etwa 1905 durchaus angesehen und einflussreich, wenn auch nicht unumstritten. Als ihre Stärken wurden die reichhaltige und genaue Dokumentation sowie eben ein hohes $\mathrm{Ma} ß$ an fruchtbarer Interdisziplinarität geschätzt. Letzteres galt nicht bloß für die deklarierten Querbezüge zwischen Ethnographie und Anthropologie, sondern auch für die starken Wechselverbindungen der Ethnographie mit Geschichte und Frühgeschichte (Archäologie) sowie mit den Sprachwissenschaften. Im letzten Viertel des 19. Jh. hatte der aus Minden in Westfalen gebürtige (und mit einer Landarzttochter aus Spital/Phyrn verheiratete) Franz Boas, ein ehemaliger Mitarbeiter von Bastian und Virchow am Berliner Museum, genau diese interdisziplinären Stärken und ihre damals noch liberalere Ausrichtung bei seiner Übersiedlung in die USA mit transportiert. ${ }^{16}$ Als einer der heute noch hoch verehrten Gründerväter der US-amerikanischen Anthropology legte Boas damit das Fundament für den in den USA quer durch das 20. Jh. hinweg maßgeblichen „Four Field Approach“, der physische, linguistische, archäologische und sozio-kulturelle Anthropologie auf kulturrelativistisch-empirischer Grundlage miteinander kombiniert. Die Ausbildung von „Folklore Studies“/Volkskunde betrieben Boas und seine zahllosen Schülerinnen und Schüler hingegen nur zögerlich, sie blieb weitgehend als Unterdisziplin in der Kulturanthropologie integriert und konnte sich als eigenes Fach ebenso wenig in den USA wie in Westeuropa durchsetzen.

Demgegenüber waren bereits lange vor Kriegsausbruch die Abgrenzungen zwischen den britischen und frankophonen Varianten von Ethnographie und Anthropologie zu jenen des deutschsprachigen Raumes weit ausgeprägter. Dies hing nicht nur mit anderen ideengeschichtlichen Traditionen zusammen, sondern auch mit handfester kolonialpolitischer Pragmatik. Die Ethnographie in Frankreich und in Großbritannien hatte zuerst ähnlich wie in den USA eine längere evolutionistische Phase durchlaufen, was in der deutschsprachigen Ethnographie - sieht man vom akademischen Außenseiter Karl Marx und seinen Anhänger/inne/n einmal $\mathrm{ab}$ - kaum eine Rolle spielte. In dem Maße, in dem sich die evolutionistischen Modelle jedoch zunehmend als zu weitmaschig für sozio-kulturelle Fragen erwiesen, wandte man sich in Frankreich und im Vereinigten Königreich ab 1900 ganz anderen Alternativen zu als jener des von Boas in den USA betriebenen „Four Field Approach“. Diese Alternativen wurden in Nordwest-Europa zunehmend in einer gegenwartsbezogenen und daher sozialwissenschaftlichen Ausrichtung der Ethnographie gesehen, wobei ab 1910 der Einfluss des großen Werkes von Emil Durkheim da wie dort maßgeblich wurde. ${ }^{17}$ Die physische Anthropologie hingegen wurde von der Ethnographie nahezu gänzlich abgetrennt und radikal in die Naturwissenschaften integriert, mit verbleibenden Querbezügen zu Urgeschichte und Archäologie. Aus dieser im Vorfeld und während des Ersten Weltkriegs erwachsenen starken Integration der Ethnographie in die Sozialwissenschaften, und ihrer systematischen Ablösung von den Naturwissenschaften erwuchs die spezifisch nordwesteuropäische Version einer auch heute noch so genannten Sozialanthropologie - welche sich im 21. Jh. freilich nicht mehr den historischen Wissenschaften verweigern kann, wie dies in der ersten Hälfte des 20. Jh. noch durchwegs der Fall war. Aus britischen und frankophonen Perspektiven der Jahre vor 1914 war die deutschsprachige Ethnographie daher weit stärker als sie selbst geschichtlich, linguistisch und naturwissenschaftlich ausgerichtet, sie hatte zu wenig Gegenwartsbezug, und sie war allzu intensiv auf materielle Kultur und auf deren Präsentation in Museen hin orientiert. Die wechselseitigen Verflechtungen waren allerdings unbestritten. Der frühe Einfluss der

\footnotetext{
${ }^{15}$ Ruggendorfer, Peter und Szemethy, Hubert D.: Felix von Luschan (1854-1924): Leben und Wirken eines Universalgelehrten, Wien: Böhlau 2009; zu Thurnwald siehe: Мецк-Косн Marion: Auf der Suche nach der menschlichen Gesellschaft: Richard Thurnwald, Berlin: Reimer 1989; zu Pöch siehe Ballhausen et al.: Archivhorizonte: Wien: Löcker i.Dr.

${ }^{16}$ Cole, Douglas: Franz Boas: The early years. 1858-1906. University of Washington Press: London - Seattle 1999; GiRTLER, Roland: Franz Boas, Burschenschaftler und Schwiegersohn eines österreichischen Revolutionärs von 1848, Anthropos 96/2001, 572.

${ }^{17}$ PARKIN in Barth, Fredrik et al. 2005, 170-185.
} 
Schriften von Vater und Sohn Forster und jener der Werke des Leipziger Museumsmannes Gustav Klemm auf die Entstehung der britischen Anthropologie waren anerkannt, ebenso wie die Anregungen, die Emil Durkheim bei seinen Deutschland-Aufenthalten dort erhalten hatte. Wechselseitige Kongressbesuche und Rezensionen waren die Regel und nicht die Ausnahme.

Regionale und quasi-nationale, teilweise aber auch eher sprachräumliche Unterschiede hatten sich also innerhalb der euro-amerikanischen Wissenschaftslandschaften von Ethnographie und Anthropologie bereits lange vor 1914 herausgebildet. Dennoch aber kann bis knapp vor Kriegsausbruch, gemeinsam mit Autoren wie Chris Manias, ${ }^{18}$ von einem Anhalten des „wissenschaftlichen Internationalismus“ durchaus weiter gesprochen werden. Die unterschiedlichen Forschungsansätze des nordamerikanischen, frankophonen, britischen und deutschsprachigen Raumes rivalisierten heftig miteinander ebenso wie untereinander, aber dies erfolgte weiterhin im Modus des akademischen Normalbetriebes und schloss unbedingt die wechselseitige Kenntnisnahme, Kommunikation und durchaus auch den akademischen Austausch mit ein.

\section{ABSCHOTTUNG UND NEUAUSRICHTUNG: DER KRIEGSEINSATZ VON ETHNOGRAPHIE UND ANTHROPOLOGIE IM DEUTSCHSPRACHIGEN RAUM}

Felix von Luschan weilte gerade auf einem Kongress in Australien, als die Regierung in Wien Serbien den Krieg erklärte, in den bald darauf auch England eintrat - was von Luschan unversehens zum Bürger eines Kriegsgegners im Feindesland machte. Die Heimreise trat er über die noch neutralen USA an, wo er mit seinem alten Freund Franz Boas zusammentraf und etliche Vorträge hielt, bevor er nach Europa übersetzte und schließlich wieder in Berlin eintraf. ${ }^{19}$ - Der aus Krakau stammende und mit Sigmund Freud befreundete Bronislaw Malinowski - heute eine Ikone der Fachgeschichte - war ebenfalls noch Inhaber von Reisedokumenten und Ausweisen der K.u.K. Monarchie, während er mit seinen Feldforschungen auf den von Australien aus verwalteten Trobriand-Inseln begann. Auch ihn machte der Kriegsausbruch zum Angehörigen eines Kriegsgegners, der allerdings von den australischen Behörden bald die Erlaubnis erhielt, seine angeordnete Internierung als beaufsichtigte Feldforschung auf diesen entlegenen Inseln fort zu setzen. Aus diesen speziellen Rahmenbedingungen erwuchs die in dieser Systematik vollkommen neue Methode der mindestens ein Jahr lang währenden ethnographischen Feldforschung unter Einheimischen - ein Prinzip, das die Kultur- und Sozialwissenschaften bis heute revolutioniert hat. Dies gilt nicht nur für die britische Social Anthropology, in der Malinowski ab diesem Zeitpunkt seinen raschen Aufstieg zu Weltruhm fortsetzte. ${ }^{20}$

Die beiden biographischen Momentaufnahmen zu den Aktivitäten von Malinowski und von Luschan im August 1914 illustrieren zweierlei: Zum einen zeigen sie den abrupten Einschnitt an, den der Kriegsausbruch überall auf der Welt für den Wissenschaftsbetrieb bedeutete, zumal in Ethnographie und Anthropologie mit ihrem hohen Anteil an grenzüberschreitender Vernetzung und an Auslandsreisen. Zum anderen wird damit aber auch auf die unterschiedlichen Richtungen verwiesen, welche Ethnographie und Anthropologie ab nun im deutschsprachigen Raum gegenüber den meisten anderen Zentren des Wissenschaftsbetriebes nahmen. Tendenziell verabschiedete sich der deutschsprachige akademische Betrieb zunehmend aus seiner bis dahin gegebenen, betont internationalen Einbindung, und schottete sich zwangläufig immer mehr auf den Bereich der im Krieg verbündeten Mächte ab. Der Ausbruch des Ersten Weltkrieges markiert in Ethnographie und Anthropologie des deutschsprachigen Raumes somit auf besonders akzentuierte Weise tatsächlich das definitive Ende des wissenschaftlichen Internationalismus im weiteren Sinn, der bis dahin - mit oder ohne liberale Vorzeichen - im Prinzip noch vorgeherrscht hatte.

Gegenläufige Tendenzen hatte es bereits seit längerem nicht nur in der Politik gegeben, sondern auch im deutschsprachigen ethnographisch-anthropologischen Wissenschaftsbetrieb selbst. Für diese Gegenströmungen zu dem vor 1914 bereits schwächer werdenden wissenschaftlichen Internationalismus

\footnotetext{
${ }^{18}$ MAnIAS, Chris: The Race Prussienne Controversy: Scientific Internationalism and the Nation, in: Isis 100 (2009), $733-757$.

19 Evans, Andrew D.: Science behind the Lines: The Effects of World War I on Anthropology in Germany, in: Johler et al.: Anthropology in Wartime 2010, 115-116.

${ }^{20}$ Young, Michael W.: Malinowski: Odyssey of an Anthropologist, 1884-1920, New Haven: Yale UP 2004.
} 
lassen sich in allen Forschungsbereichen charakteristische Beispiele anführen. In der physischen Anthropologie war mit der Anwendung der Mendelschen Erblehre auf die menschliche Reproduktion durch Ernst Haeckel und seine Schüler schon kurz nach der Jahrhundertwende ein Paradigma eingeführt worden, das eine neue wissenschaftliche Theorie von menschlichen „Rassen“ zu liefern beanspruchte. ${ }^{21}$ Eine systematische Anwendung und Beweisführung im Sinn dieser neuen Theorie versuchte Eugen Fischer in seinem Buch von 1913 über die sogenannten Bastards von Rehobot, das auf seinen Untersuchungen in der damaligen Kolonie „Deutsch Südwest-Afrika“ (heute: Namibia) beruhte. ${ }^{22}$ Die Haeckel-Fischer'schen Ansätze markierten nicht nur eine Abkehr von der liberaleren physischen Anthropologie des einige Jahre davor verstorbenen Virchow, sondern auch eine verstärkte Binnenausrichtung. In Berlin setzte von Luschan dem uneingeschränkten Führungsanspruch des neuen Paradigmas bis an sein Lebensende zwar zeitweilig inhaltliche und auch organisatorische Schranken, konnte sich aber nie zur Ausarbeitung einer veritablen Alternative entschließen, wie sie zeitgleich etwa von Franz Boas in den USA verfolgt wurde. All dies bedeutete freilich nicht, dass die neue Rassentheorie bereits eine offen rassistische Politik befürwortet oder gefordert hätte. Dies war in Bezug auf die deutschen Kolonien auch gar nicht notwendig, da eine solche dort längst praktiziert wurde. Umgekehrt waren wissenschaftliche Rassentheorien in jenen Jahren kein deutschsprachiges Unikum, sondern existierten in Teilbereichen ebenso auch in der englisch- und französischsprachigen Anthropologie. Allerdings kann nicht übersehen werden, dass derselbe Eugen Fischer, der die methodische Wende vor 1914 einleitete, ab 1933 ein besonders einflussreicher Akteur in seinem Fach und auch in dessen verhängnisvoller Verstrickung mit NS-Verbrechen werden sollte.

In der deutschen Völkerkunde hatte sich etwa um die Zeit von Bastians Ableben ein Teil seiner ehemaligen Schüler und Mitarbeiter unter Gräbner und Anckermann vom geistigen Erbe ihres Lehrers losgesagt, um in der Nachfolge des Anthropogeographen Friedrich Ratzel die Grundlagen der sogenannten „Kulturkreislehre“ zu propagieren. In ihrer säkularen Variante postulierte dieser Ansatz die Ausbreitung von Ideen aus wenigen Zentren als historisches Movens, und vertrat damit zugleich ein Genie-orientiertes Weltbild, das einher ging mit der Vorstellung von der generellen „Ideenarmut“ der Menschheit. Während Leo Frobenius seine eigene phänomenologische Richtung am Rande der Genie-orientierten Version dieser Theorie weiterentwickelte, wurde in Wien vom westdeutschen Pater Wilhelm Schmidt und seinen Schülern die katholische Version der Kulturkreislehre ausgearbeitet und zugleich eine erbitterte, innerkirchliche Gegnerschaft zum liberalen tschechischen Priester Alois Musil aufgebaut. ${ }^{23}$ In diesen verschieden akzentuierten säkularen, theologischen und später auch biologistischen Varianten sollte die Kulturkreislehre weite Bereiche von Kunst- und Kulturgeschichte des deutschsprachigen Raums über ein halbes Jahrhundert hinweg deutlich dominieren, während sie anderswo in der weltweiten Kultur- und Sozialanthropologie auf wenig bis keinerlei Resonanz stieß. Damit war auch in einigen wichtigen Teilbereichen der deutschsprachigen Völkerkunde in ersten Ansätzen bereits vor 1914 ein zunehmend isolationistischer Sonderweg eingeschlagen worden, der sich während und nach dem Ersten Weltkrieg verstärken sollte.

Die unvermeidliche organisatorische und intellektuelle Abgrenzung gegenüber der akademischen Welt im Bereich der Kriegsgegner verstärkte quasi „von oben nach unten“ die eher isolationistischen theoretisch-methodischen Tendenzen der deutschsprachigen Ethnographie und Anthropologie. Zugleich ist zu vergegenwärtigen, dass der Kriegsausbruch 1914 speziell in Österreich-Ungarn und Deutschland „,von unten nach oben" von einem beispiellosen Enthusiasmus der Massen mitgetragen war, dem sich auch auf der politischen Ebene keine der großen Parteien verschloss. ${ }^{24}$ Daher ist zumindest als Arbeitshypothese davon auszugehen, dass auch ein Großteil der Ethnologen und Anthropologen als loyale Staatsbeamte, die die Mehrheit unter ihnen waren, die Kriegserklärungen ihrer eigenen Regierungen mehr oder minder aktiv

\footnotetext{
${ }^{21}$ Evans, Andrew D.: World War I and the Science of Race in Germany. Chicago: Chicago University Press 2010.

22 GessLer, Bernhard: Eugen Fischer (1874-1967). Leben und Werk des Freiburger Anatomen, Anthropologen und Rassenhygienikers bis 1927. Frankfurt/Main: Peter Lang Verlag 2000; Loesch, Niels C.: Rasse als Konstrukt. Leben und Werk Eugen Fischers. Frankfurt/Main: Peter Lang Verlag 1997.

${ }^{23}$ Gingrich, Andre und Steger, Brigitte: Kulturkreis, in: Kreff, Fernand, Knoll, Eva-Maria, Gingrich, Andre (Hg.): Lexikon zur Globalisierung: Anthropologische und sozialwissenschaftliche Zugänge zur Praxis, Bielefeld: transcript 2011, pp. 217-219.

${ }^{24}$ Rauchensteiner, Manfried: Der Tod des Doppeladlers: Österreich-Ungarn und der Erste Weltkrieg, 2. Auflage Graz: Steirische Verlagsgesellschaft 1998.
} 
unterstützten. Dies markiert einige der wesentlichen Rahmenbedingungen, unter denen der einzigartig intensive Kriegseinsatz der deutschsprachigen Ethnographie und Anthropologie zustande kam. In seiner Stoßrichtung und in seinen Ausmaßen kann ihm für die Zeit des Ersten Weltkriegs keinerlei auf irgendeine Weise vergleichbare Parallele auf der Seite der Kriegsfeinde gegenübergestellt werden. Der Kriegseinsatz der deutschsprachigen Ethnographie und Anthropologie hatte mehrere Hauptbestandteile, und umfasste a) Spionage und Propaganda, b) wissenschaftliche Expeditionen in Zielgebieten der Kriegsführung sowie c) Untersuchungen im Kriegsgefangenen-Lagern. Gemeint sind hier nur jene Aktivitäten, die von Wissenschaftern gesetzt wurden, die beruflich ganz oder wenigstens teilweise als Ethnologen oder Anthropologen tätig waren. Nicht angesprochen ist hier also jene Vielzahl an anderen Dokumente und Quellen von besonderem ethnographischem Gehalt, die von Armee-Angehörigen stammen, die beruflich nicht als Ethnographen oder Anthropologen tätig waren etwa die Berichte von K.u.K. Armee-Angehörigen aus Ostanatolien und Mesopotamien. ${ }^{25}$

Spionage und Propaganda als Aufgabengebiet der deutschsprachigen Ethnographie und Anthropologie im Ersten Weltkrieg umfasste den kleinsten dieser drei Teilbereiche, und zugleich den einzigen der in gewissem Ausmaß auch auf der Seite der Kriegsgegner betrieben wurde, wie man heute weiß. ${ }^{26}$ Die Beispiele beziehen sich stets auf Einzelpersonen mit sehr unterschiedlicher Kompetenz. Als militärisch effizientester Kundschafter für das Haus Habsburg im Nahen Osten kann der böhmische Priester, Orientalist und Ethnograph Alois Musil angesehen werden, der „Lawrence of Moravia“, wie ihn Ernest Gellner respektvoll bezeichnete. ${ }^{27}$ In seinem Fall wurde wirksam, dass ethnographische Feldforschung bei mitteleuropäischen Experten vor 1914 in vielen Fällen bereits sehr stark ausgebildet war. Musil - ein Cousin des Dichters Robert Musil, später auch ein Beichtvater von Kaiserin Zita - vertrat eine ausgesprochen moderne Form der Ethnographie und dürfte auch nachrichtendienstlich relativ erfolgreich gewesen sein. Hingegen erwies sich die Kundschafter-Tätigkeit von Leo Frobenius in Ostafrika eher als weitgehend nutzlos. Knapp vor Kriegsende versuchte der später weltberühmte deutsche Afrika-Experte auf wenig erfolgreiche Weise geeignete Operationen einheimischer Gruppen in die Wege zu leiten, um gegnerische Kräfte zu binden. ${ }^{28}$

Unter den wissenschaftlichen Expeditionen ist in erster Linie die Ethnographische Albanien-Expedition zu nennen, welche unter Leitung von Michael Haberlandt 1917 von Bosnien aus in enger Zusammenarbeit mit der K.u.K. Armee durchgeführt wurde. ${ }^{29}$ Albanien war bereits seit dem letzten Viertel des 19. Jh. ein ausgesprochenes Interessen- und Spezialgebiet besonders für österreichische Experten geworden, einschließlich Sprachwissenschaften und Ethnographie. Der Verlauf der militärischen Kräfteverhältnisse in den letzten beiden Kriegsjahren brachte es mit sich, dass der Teilrückzug des italienischen Gegners der K.u.K. Armee die Chance auf eine Wende durch verstärkte eigene Präsenz auf diesem Teil des Balkan zu eröffnen schien. Die Ethnographische Balkan-Expedition erfüllte in mancher Hinsicht durchaus die selbstgesteckten Ziele der verbesserten Dokumentation und der zusammenfassenden Einsichten zu dieser Region; hier dürfte auch das Verhältnis zwischen militärischem Aufwand (v.a. bei Logistik oder Sicherung) und militärischem Nutzen (verbessertes Wissen in Teilbereichen) relativ ausgewogen ausgefallen sein.

Der dritte und bei weitem umfangreichste Hauptbestandteil des ethnographisch-anthropologischen Kriegseinsatzes in Deutschland und Österreich-Ungarn betrifft die Untersuchungen in den Kriegsgefangenen-

25 SiX-Hohenbalken, Maria: “A moral witness within complicity” - Edmund Jaroljmeks Tagebuchaufzeichnungen aus Mosul 1916. Ein Beitrag zur Anthropologie von Gewalt, in: Anthropos 107/2012: 71-86.

${ }^{26}$ PrICE, David H.: Anthropological Intelligence. The Deployment and Neglect of American Anthropology in the Second World War, Durham - London: Duke University Press 2008.

27 BAuer, Karl J.: Alois Musil: Wahrheitssucher in der Wüste, Wien: Böhlau 1989; Gellner, Ernest: Anthropology and Politics: Revolutions in the Sacred Grove, Oxford: Blackwell-Wiley 1995, 212-228; JunG, Peter: Der k.u.k. Wüstenkrieg: ÖsterreichUngarn im Vorderen Orient, 1915-1918, Graz: Styria: Militärhistor. Dienst, 1992; siehe auch FeIGL, Erich: Musil von Arabien: Vorkämpfer der islamischen Welt, Frankfurt/M.: Ullstein 1988.

${ }^{28}$ Zitelmann, Thomas: Des Teufels Lustgarten: Themen und Tabus der politischen Anthropologie Nordostafrikas. Habil.schrift FU Berlin 1999; BRAUKÄMPER, Ulrich: Im Spannungsfeld zwischen Wissenschaft und politischem Aktivismus: Leo Frobenius als Geheimagent in Nordost-Afrika, in: KoHL, Karl-Heinz und Platte, Editha (Hg.): Gestalter und Gestalten: 100 Jahre Ethnologie in Frankfurt am Main, Frankfurt - Basel: Stroemfeld, 167-186.

${ }^{29}$ Marchetti, Christian: Austro-Hungarian Volkskunde at War: Scientists on Ethnographic Mission in World War I, in Johler et al.: Anthropology in Wartime 2010, 207-230. 
Lagern. Diese Lageruntersuchungen wurden in Österreich von Rudolph Pöch und in Deutschland von seinem früheren Lehrer Felix von Luschan initiiert und koordiniert, wobei die maßgeblichen Förderinstitutionen das Berliner Museum für Völkerkunde und die Wiener Akademie der Wissenschaften waren; auch die Akademie der Wissenschaften in Budapest führte in geringerem Ausmaß derartige Erhebungen durch. ${ }^{30}$ Teilweise kooperierten die Berliner, Wiener und Budapester Wissenschaftergruppen auch vor Ort miteinander, insgesamt war der österreichische Anteil an den Erhebungen in diesen Lagern aber deutlich der höchste. Das Programm der Lageruntersuchungen umfasste durchwegs sowohl physisch-anthropologische als auch ethnographische Dimensionen. In der Praxis wurde allerdings weit eher die physisch-anthropologische Seite der Erhebung relativ konsequent umgesetzt (dies noch deutlicher von Wien als von Berlin aus), während die ethnographische Seite in der Praxis nur teilweise (in Wien: später) und eher bruchstückhaft verwirklicht wurde.

In allen drei genannten Bereichen von wesentlichen ethnographisch-anthropologischen Kriegsaktivitäten in Deutschland und Österreich waren Kooperationen seitens der beteiligten Wissenschafter und ihrer jeweiligen institutionellen Leitung mit der Armeeführung sowie mit den befassten Truppenteilen unumgänglich. Allerdings zeigt eine nähere Analyse, dass die jeweiligen Interessenslagen zwischen Wissenschaft und Armee dabei in jedem dieser drei Bereiche recht unterschiedlich ausgeformt waren. In den eher seltenen Fällen von aktiver nachrichtendienstlicher Tätigkeit, wofür die Beispiele von Musil und Frobenius angeführt wurden, musste die Armee ein aktives und positives Interesse an der erfolgreichen Durchführung von guter Spionage in exponierten Frontregionen wie Nordarabien und Ostafrika haben, und dieses auch aktiv bei den potenziell dafür geeigneten Wissenschaftern mit entsprechender Felderfahrung artikulieren. Bei der Albanien-Expedition unter Leitung von Haberlandt hingegen scheinen die vorliegenden Quellen auf eine etwas zurückhaltendere KostenNutzen-Kalkulation seitens der Armee hinzuweisen. Im administrativ und finanziell wohl aufwendigsten Fall der Lageruntersuchungen schließlich weist alles darauf hin, dass die Armeeführung relativ skeptisch blieb über Sinn und Zweck dieser Untersuchungen. Hierbei handelte es sich nahezu ausschließlich um eine aktive Initiative der betreffenden Wissenschafter, die auf keinerlei Beauftragung durch die Armee zurückging, sondern von der die Armeeführung zum Teil erst recht mühsam überzeugt werden musste. ${ }^{31}$ Daraus ergibt sich die Frage nach den Motiven der Wissenschafter für diese spezielle Form der Untersuchungen, denen in Friedenszeiten keine direkten Vorläufer vorangegangen waren, und denen nun in Kriegszeiten auch keinerlei Parallele auf der Seite der Kriegsgegner entsprach. Da die deutsch-österreichischen Untersuchungen in Kriegsgefangenen-Lagern folglich das eigentliche Moment an praktischer Veränderung und „Transformation“ in Ethnographie und Anthropologie während der Kriegszeit darstellten, ist es umso wesentlicher, die Motive für diese Transformation zu identifizieren.

Drei Bündel an Motiven lassen sich dabei unterscheiden, die alle in dieselbe Richtung wiesen und deren Kombination ausschlaggebend gewesen sein dürfte. Erstens, Ethnographie und Anthropologie in Deutschland und Österreich hatten schon lange vor 1914 immer wieder auf vielfältige Weise mit Armee-Gliederungen zusammen gearbeitet. So waren schon einige der großen Expeditionen (wie die Berliner Sepik-Expedition 1912/13, und die K.u.K. Südarabien-Expedition 1898) mit partieller Unterstützung der Marine zustande gekommen. ${ }^{32}$ Wollte man das eigene Fach auch in Kriegszeiten festigen, so war es nach 1914 in Deutschland und Österreich umso eher naheliegend, an diesen etablierten Kooperationserfahrungen an zu knüpfen. Zweitens, Forschungen im Armee-nahen Bereich versprachen Ethnographie und Anthropologie nach 1914 eine Reihe logistischer Vorteile. Dazu zählten im Vergleich zu zivilen Alternativen der Forschung die bei entsprechender Nähe zum Militär weit besseren Aussichten auf Bewilligungen und Finanzierungen, auf eigenen Schutz und bei entsprechender Argumentation auch auf Befreiung vom Fronteinsatz wegen der „Kriegswichtigkeit“ dieser Untersuchungen. Drittens gingen die maßgeblichen Ethnographen und Anthropologen davon aus, dass Untersuchungen in den Kriegsgefangenenlagern ähnliche Chancen und Möglichkeiten bieten würden wie klassische Feldforschungen im Feld, das meist in der Ferne lag. Der Nachteil, dass die Forschungssituation in

\footnotetext{
${ }^{30}$ Turda, Marius: The Idea of National Superiority in Central Europe, 1880-1918. New York: Edwin Mellen Press 2005.

31 Gingrich, Andre: After the Great War 2010, 371.

32 Geisenhainer, Katja: „Rasse ist Schicksal.“ Otto Reche (1879-1966) - ein Leben als Anthropologe und Völkerkundler. Beiträge zur Leipziger Universitäts- und Wissenschaftsgeschichte, Reihe A Band 1, Leipzig: Evangelische Verlagsanstalt 2002; JANATA, Alfred: Jemen - Im Land der Königin von Saba. Wien: Museum für Völkerkunde 1989.
} 
den Lagern Veränderungen und Einschränkungen gegenüber der Feldforschung ,,in situ“ in der Ferne mit sich bringen würde, wäre aufgewogen durch die Tatsache, dass die Erhebungen ,ex situ“ in den nahe gelegenen Lagern in weit größerem quantitativem Umfang stattfinden könnten, und zugleich billiger und rascher vor sich gehen würden.

Als eigentliches Kernmotiv für die ethnographischen und anthropologischen Forschungen in den Kriegsgefangenen-Lagern Deutschlands und Österreich-Ungarns lässt sich damit der Wunsch nach Nutzung der scheinbar immens verbesserten Forschungsbedingungen identifizieren, die im plötzlich massenhaften Vorhandensein in Mitteleuropa von gefangenen Menschen aus weit entlegenen Ländern erblickt wurden. Statt unsichere Expeditionen in weit entfernte Gebiete wagen zu müssen, könne man nun die viel überschaubarere und besser kontrollierte Situation in den Lagern nutzen - auf diesen einfachen Nenner kann das Grundmotiv herunter gebrochen werden. Gegenüber den militärisch damit befassten Stellen und Behörden wurde diese Interessenslage der Wissenschaften durchaus dargelegt, aber in Verbindung mit dem Argument, dass derartige Kriegsgefangenen-Forschungen durch Ethnographie und Anthropologie der Armee ebenfalls wertvolle Einsichten vermitteln könnten, nämlich über die physische und kulturelle Beschaffenheit und Wehrhaftigkeit der feindlichen Armeen im Krieg. ${ }^{33}$ De facto erwiesen sich diese optimistischen Behauptungen als haltlos; in militärischer Hinsicht hatten die Kriegsgefangenen-Forschungen keinerlei substanziellen Nutzen - was bereits eine Teilantwort auf die eingangs gestellten beiden Fragen darstellt.

Die praktische und methodische Durchführung und Umsetzung der Kriegsgefangenen-Forschungen brachte die eigentliche, grundsätzliche Transformation von Ethnographie und Anthropologie während des Ersten Weltkriegs mit sich. Daraus ergaben sich nachhaltige Folgen; ihre Diskussion bildet daher den Abschluss dieses Abschnittes. Voran zu schicken ist dem allerdings, dass es eben nicht die Erarbeitung irgendwelcher kurzfristigen Ergebnisse, Einsichten, Entdeckungen oder Theorien war, die vor Kriegsende aus den Lagerforschungen resultiert wäre. Im Gegenteil, die Materialien der Kriegsgefangenen-Forschungen erbrachten eine derartige Flut an Daten, dass die Forscher kaum mit ihrer systematischen Anlage nach gekommen sein dürften und häufig betonten, dass die Auswertung auf die Zeit nach Kriegsende warten müsse. Es war also die Art und Weise der Organisation der Erhebungen selbst, welche die eigentliche, grundsätzliche Veränderung für Ethnographie und Anthropologie mit sich brachte. ${ }^{34}$

In methodischer, organisatorischer und technischer Hinsicht lag der grundlegende Unterschied der Kriegsgefangenen-Forschungen $\mathrm{zu}$ den anthropologisch-ethnographischen Erhebungen der Friedenszeiten vor 1914 zunächst tatsächlich in ihren quantitativen Ausmaßen, und in Verbindung damit in der vergleichsweise größeren zeitlichen Geschwindigkeit, mit der diese größeren Datenmengen generiert wurden. Die Kriegsgefangenen-Lager in Wünsdorf bei Berlin, in Ostösterreich, Ungarn (Eger) und in Böhmen (Theresienstadt) waren für die Forscher verkehrsmäßig leicht zugänglich, und meist genügten Aufenthalte von wenigen Tagen oder Wochen zur Durchführung ganzer Kampagnen von Datenerhebungen. Diese wurden in Kooperation mit der jeweiligen Lagerleitung und zumeist im Team durchgeführt - anfangs häufiger unter Leitung der jeweils zuständigen Hauptverantwortlichen, später immer häufiger auch durch die jüngeren Assistenten und spezialisierten Mitarbeiter von Pöch (vor allem Josef Weninger) und von Luschan.

Diesem grundlegenden quantitativen und zeitlichen Faktor war auch eine wichtige personelle Dimension zu eigen, die entscheidende Langzeit-Wirkungen beinhaltete. Sie bestand im Charakter der KriegsgefangenenStudien als großer Ausbildungsstätte für den wissenschaftlichen Nachwuchs, und für Kollegen, die bis dahin wenig oder kaum irgendeine empirische Praxis zu sammeln Gelegenheit erhalten hatten. Eine ganze Generation von Schülern Pöchs und von Luschans wurde so relativ dicht und intensiv in - aus heutiger Sicht durchaus fragwürdige - Methoden der ethnographisch-anthropologischen Erhebungen eingeführt und darin geschult. Meiner bisherigen provisorischen Schätzung zufolge kann davon ausgegangen werden, dass etwa ein Viertel

${ }^{33}$ Evans, Andrew: Science behind the Lines, in Johler et al.: Anthropology in Wartime 2010, 115; Berner, Margit: Large-Scale Anthropological Surveys in Austria-Hungary, 1871-1918, in: Johler et al.: Anthropology in Wartime 2010, 248-252; ScHEER, Monique: Captive Voices: Phonographic Recordings in the German and Austrian Prisoner-of-War Camps of World War I, in: Johler et al.: Anthropology in Wartime 2010, 287-308.

${ }^{34}$ LANGe, Britta: AfterMath, in Johler et al.: Anthropology in Wartime 2010, 312-333. 
bis ein Drittel aller Ethnographen und Anthropologen des deutschsprachigen Raums jener Zeit (die das Fach mehr oder minder berufsmäßig betrieben, oder die knapp vor ihren Studienabschlüssen standen) direkt oder indirekt eingebunden waren in die Kriegsgefangenen-Forschungen. Ganze Karrieren wurden so entweder weiter befördert oder überhaupt auf ihre Wege geschickt. Nicht unwesentlich für die weitere Geschichte von Ethnographie und Anthropologie ist dabei, dass ein Großteil jener Anthropologen (und ein kleinerer Teil der Ethnographen), die nach 1933/38 in NS-Deutschland einflussreich werden sollten, ihre entscheidenden empirischen Ausbildungsschübe in den Lagererhebungen 1914-18 erhielten. Zu ihnen zählten auch die später prominenten physischen Anthropologen und „Rassenkundler“ Otto Reche und Egon von Eickstädt. ${ }^{35}$

Eine wichtige qualitative Bestimmung der speziellen Forschungssituation in den Kriegsgefangenenlagern bestand selbstverständlich darin, dass sich die untersuchten Personen von vorne herein in einer abhängigen Zwangssituation befanden - direkt gegenüber der Lagerleitung und den entsprechenden administrativen und militärischen Diensträngen, indirekt aber auch gegenüber den Forschern als Angehörigen und Vertretern jener Macht, welche die Kriegsgefangenen in den Lagern festhielt. Der Status als unfreier Gefangener machte somit einen gravierenden Unterschied aus im Vergleich zu Erhebungen in Friedenszeiten, bei denen die untersuchten Personen selbst unter Bedingungen kolonialer Fremdherrschaft als „abhängige Freie“ normalerweise weit größeren Spielraum für die Entscheidung hatten, ob sie sich an einer ethnographischen oder anthropologischen Untersuchung beteiligen wollten oder nicht. Auch in den Lagern des Ersten Weltkriegs stand den Kriegsgefangenen die Entscheidung zwar grundsätzlich frei, ob sie an den Untersuchungen mitmachen wollten oder nicht - aber der Spielraum dafür war wesentlich enger, und die Hierarchie zwischen Gefangenem und Wissenschafter war von vornherein weitaus markanter. Die Lagerleitungen und die Wissenschafter mussten dennoch einigen Aufwand betreiben - der von kleinen Geschenken (etwa: Zigaretten) bis zum beispielhaften Vorführen eines Gipsabdruckes reichte - um Gefangene zur Mitwirkung zu gewinnen. ${ }^{36}$

Der systematische Einsatz wissenschaftlicher Dokumentationsmittel und -geräte ist ein markanter Unterschied, den die groß angelegten Lagerforschungen gegenüber der Vielfalt von Erhebungsformen aufwiesen, die vor Beginn des Ersten Weltkriegs vorgeherrscht hatten. Photographische und kinematographische Aufnahmen, Tondokumentationen sprachlicher oder musikalischer Art, Abgipsen und Vermessen sowie die entsprechenden, durch formalisierte Erhebungsbögen vorstrukturierten schriftlichen Aufzeichnungen und Übersetzungen waren zwar alle schon vor dem Ersten Weltkrieg in anthropologischen und ethnographischen Erhebungen zum Einsatz gekommen, aber nur unregelmäßig. Vor 1914 waren nämlich jene großen Expeditionen eher die seltene Ausnahme gewesen, bei denen diese Gerätschaften und Techniken wirklich systematisch verwendbar gewesen wären. Die Mehrheit der vor dem Krieg vorwiegenden Erhebungen waren durch Einzelpersonen oder Kleingruppen durchgeführt worden, was diesen eher nur sporadischen und selteneren Einsatz bedingt hatte. ${ }^{37}$ Die Initiierung der Kriegsgefangenenlager-Forschungen änderte dieses Verhältnis von Grund auf. Ethnographisch-anthropologische Einzelforschungen waren nun die seltene Ausnahme, während die breit angelegten Lagerforschungen als Großprojekte nach innen und außen den gültigen Standard setzten. Der massive Einsatz der nach damaligem Entwicklungsstand modernsten Art von Gerätschaft, Technik, und Dokumentationsinstrumentarien folgte dabei den naturwissenschaftlichen Idealen, welche auch in Ethnographie und Anthropologie als damals noch in die Naturwissenschaften integrierten Fächern Geltung beanspruchten. Zugleich war - durchaus nachvollziehbar - ein wesentlicher Zweck dieses breiten Einsatzes von Gerätschaften und Techniken die Standardisierung der Erhebung, also das Bestreben, vergleichbare und auf dieselbe Art und Weise gewonnene Daten im Rahmen dieser Großprojekte zu generieren.

Eine bereits in der anfänglichen Konzeption der Lagerforschungen versteckt angelegte Tendenz erhielt durch diese Umsetzung eine zusätzliche, irreversible Dynamik. Im Ergebnis führte sie zu einer kolossalen Bevorzugung und Aufwertung der physisch-anthropologischen Forschungen, bei gleichzeitiger Marginalisierung und Reduzierung des Stellenwerts der Ethnographie. Wie angedeutet, war diese Tendenz

\footnotetext{
${ }^{35}$ Evans, Andrew: Science behind the Lines, in Johler et al.: Anthropology in Wartime 2010, 115; GeisenhaIner, Katja: "Rasse ist Schicksal", 2002; LANGE, Britta: AfterMath, in Johler et al.: Anthropology in Wartime 2010, 319-323.

${ }^{36}$ Lange, Britta und Gingrich, Andre: Wünsdorf-Reise 1917, in: Ballhausen et al. (i.Dr.).

${ }^{37}$ GINGRICH, Andre: Liberalism in imperial anthropology: Notes on an implicit paradigm in continental European anthropology before World War I, in: Ab Imperio 1/2007, 224-239.
} 
bereits angelegt in den frühen schriftlichen Konzeptionen und Anträgen von Pöch und von Luschan, in denen deutlich ausführlicher von physisch-anthropologischen Vermessungen und Abgüssen die Rede war als von ethnographischen Dokumentationen. Dies hatte nicht nur mit dem für Militärs eher argumentierbaren und nachvollziehbaren Charakter der physischen Anthropologie zu tun, von deren Ergebnissen gewisse Einsichten in Gesundheit und physische Wehrkraft der Kriegsgegner erwartet werden konnten. ${ }^{38}$ Darüber hinaus war diese Tendenz zur Aufwertung der physischen Anthropologie aber auch ursächlich mit den Kompetenzen und der Expertise der beiden Hauptkoordinatoren von Luschan und Pöch verknüpft: Für körperliche Vermessungen und physische Dokumentationen von Menschen egal welcher Herkunft waren sie beide ausgebildet und praktisch erfahren. Für die ethnographische Erhebung unter jener Vielzahl von Kulturen, aus denen die Mehrheit der Kriegsgefangenen stammten, fehlten ihnen fast alle erforderlichen sprachlichen und kulturwissenschaftlichen Voraussetzungen. In einem der beiden Bereiche, so wussten von Luschan und Pöch also von vorne herein, würden sie als Experten brillieren können, im anderen der beiden Bereiche hingegen drohten ihnen die Blamage, als partielle Dilettanten bloß gestellt zu werden.

Dass Pöch und von Luschan dieses eigene Defizit durchaus bewusst war, zeigt der Umstand an, dass sie sich frühzeitig bemühten, einzelne kulturwissenschaftliche Spezialisten mit ausgelagerten Subprojekten der Lagerforschungen zu beauftragen. Dies geschah etwa im Bereich der Ton- und Musikaufnahmen, für den in Wien der Musikethnologe Robert Lach durch Pöch gewonnen wurde, oder im Bereich der kaukasischen Sprachen, für den Pöch den jungen Robert Bleichsteiner (später Kustos und nach 1945 erster Direktor am Museum für Völkerkunde) einsetzte. ${ }^{39}$ Dennoch blieben diese Bemühungen eher minimalistisch angesichts der ungeheuren Vielzahl von Sprachen und Kulturen der Räume Südost- und Osteuropas sowie des Kaukasus und Zentralasien, die in den Lagern Österreich-Ungarns vertreten waren, sowie von Nord- und Westafrika, Südund Südostasiens vorwiegend in deutschen Lagern. Die dafür notwendige Expertise war zum Teil gar nicht vorhanden, zum Teil hätte sie wohl zu viel gekostet, was beides in ungünstigem Verhältnis gestanden wäre $\mathrm{zu}$ den rascheren und vergleichsweise unaufwendigeren physisch-anthropologischen Aufnahmeverfahren. Dementsprechend zufällig, fragmentarisch und künstlich in Szene gesetzt wirkt daher aus heutiger Sicht die kleinere Zahl von ethnographischen Ton-, Musik-, Bild- und Filmdokumenten aus den KriegsgefangenenLagern des Ersten Weltkriegs gegenüber der Flut von standardisierten Zahlenkolonnen und der Masse erhaltener Gipsabdrucke, die aus den physisch-anthropologischen Erhebungen resultierten.

Die praktische und organisatorische Umsetzung verschärfte und vertiefte diese Diskrepanz zwischen Ethnographie und Anthropologie noch um ein Vielfaches. Dafür kann die Logik der jeweiligen Forschungsprozesse von Ethnographie und Anthropologie als maßgebliche Ursache rekonstruiert werden. Bei einer physisch-anthropologischen Erhebung muss der Proband im wesentlichen nur still halten - ob er dies aus freien Stücken oder unter Zwang tut, ist im Kern für die Datenqualität belanglos, sondern wirkt sich nur auf den Verlauf der Datenerhebung aus, der dann mehr oder weniger kompliziert vor sich geht. Im Grunde aber setzte die physisch-anthropologische Datenerhebung der damaligen Zeit keine persönliche Interaktion voraus, im Gegenteil war diese eigentlich überflüssig. Bei einer ethnographischen Datenerhebung hingegen muss der Informant aktiv und kreativ partizipieren - und dafür sind einschüchternde Rahmenbedingungen geradezu kontraproduktiv. Als willkommene Abwechslung scheinen die ethnographischen Erhebungen nur von den wenigsten Kriegsgefangenen wahrgenommen worden zu sein. Es war also auch und nicht zuletzt die Gesamtheit der auf Mangel, Zwang und Hierarchie beruhenden, anonymisierten und unpersönlichen Erhebungsbedingungen der Gefangenenlager selber, welche die physisch-anthropologische Erhebung massiv bevorzugten und die ethnographische Erhebung an den Rand drängten. Die systematische Aufwertung der physischen Anthropologie und die gleichzeitige Marginalisierung, Abwertung und Unterordnung der Ethnographie ist somit die entscheidendste und nachhaltigste „Transformation“, welche der Erste Weltkrieg und die Involvierung beider Fachgebiete in seinem krisenhaften Verlauf für sie hervorbrachten.

\footnotetext{
${ }_{38}$ Berner, Margit: Large-Scale Anthropological Surveys in Austria-Hungary, 1871-1918, in Johler et al.: Anthropology in Wartime 2010, 233-254.

${ }^{39}$ Muehlfried, Florian: R. Bleichsteiners ,Kaukasische Forschungen', in Georgica 2002/24, 51-58.
} 


\section{ZEHN JAHRE DANACH: VERFESTIGUNG EINES SONDERWEGS UM 1928}

Die in diesem Aufsatz verfolgte Langzeit-Perspektive legt es nahe, für die Beurteilung der Folgewirkungen einen methodisch etwas unkonventionellen zeitlichen Sprung vorzunehmen. Die Periode unmittelbar nach Kriegsende mit ihren großen politischen und sozialen Umbrüchen, Friedensverträgen und versuchten Revolten ist nicht die geeignetste historische Phase, um zu verstehen, welche wissenschaftlichen Kräfte und Richtungen sich in der Zwischenkriegszeit auf Dauer durch zu setzen vermochten. Dafür bieten die späten 1920er-Jahre ein geeigneteres Umfeld an, in dem sich die Nachkriegsordnung im deutschsprachigen Raum relativ stabilisiert hatte, und in der die Folgewirkungen der innerwissenschaftlichen Transformationen von Ethnographie und Anthropologie im deutschsprachigen Raum klarer erkennbar wurden. Die Momentaufnahme der Zeit um 1928 ist daher exemplarisch anstelle eines zeitlichen Längsschnittes gewählt. Ende der 1920er-Jahre bieten jene Fächer, die vor 1914 und noch bis 1918 als „Ethnographie und Anthropologie“ bekannt waren, im deutschsprachigen Raum das Bild eines verfestigten Sonderweges, der einige Elemente westlicher und einige andere Elemente ostund nordosteuropäischer Wissenschaftsentwicklung zu einem eigenen, dritten Weg bündelt.

Im Westen - also insbesondere in den USA, in Großbritannien und in Frankreich - waren nach 1918 jene Wege in den betreffenden Fächern fortgesetzt worden, die vor 1914 bereits sehr deutlich ausgeformt waren. In den USA setzte der mittlerweile wohl etablierte Franz Boas mit seinen Schülerinnen und Schülern den kulturrelativistischen Weg seines „Four Field Approach“ fort, in dem physische Anthropologie und Kulturanthropologie zwei gleichberechtigte Zweige eines interdisziplinären Fächerbündels waren. Neben der Untersuchung einheimischer kultureller (v.a. indianischer und afro-amerikanischer) Minderheiten wurden dabei zunehmend auch Kulturen außerhalb der USA untersucht, etwa in Sibirien, Ozeanien, oder in Südamerika. Die Boas'sche Kulturanthropologie war historisch orientiert, eng mit Archäologie und Sprachwissenschaften verbunden, und pflegte weiter ihre Querbezüge zu einer physischen Anthropologie ohne Vorherrschaft des einen über das andere Fach. ${ }^{40}$ - In Großbritannien und Frankreich entwickelte sich eine Ethnologie und Sozialanthropologie weiter, die im wesentlichen ihre Grundinspirationen von Emil Durkheim erhalten hatte und daher prinzipiell sozialwissenschaftlich orientiert war. ${ }^{41}$ Die physische Anthropologie war Teil der Biologie und unterhielt kaum irgendwelche Kooperationselemente mit dieser Sozialanthropologie. Letztere hatte in jenen beiden Ländern, die sich als die Hauptsieger des Ersten Weltkrieges verstanden und die am meisten von der Neuaufteilung der kolonialen Welt nach 1918 profitiert hatten, einen relativ starken Aufschwung erfahren. Demgemäß lagen auch die Hauptaktivitäten der britischen und französischen Sozialanthropologie in den eigenen Kolonien. Das Studium der ,eigenen Kultur" gelangte hingegen in den USA, Frankreich und England bis 1939 nie über ein Schattendasein hinaus, eine „Volkskunde“ oder „Europäische Ethnologie“ als eigenes universitäres Fach blieb also im Westen de facto inexistent. In den USA spezialisierten sich dafür kaum ein halbes Dutzend von Universitätsinstituten sowie wenige Zeitschriften und akademische Verbände; in Frankreich und Großbritannien wurde nicht einmal das etabliert. Diese Aufgabe übernahmen am ehesten andere Fächer wie Kunstgeschichte, Soziologie oder Geschichtswissenschaften ${ }^{42}$

In Ost-, Nord- und Südosteuropa waren vor und nach dem Ersten Weltkrieg eine ganze Reihe von unabhängigen Ländern ohne eigene Kolonien entstanden. In diesen eben erst unabhängigen Nationalstaaten oder unabhängigen föderativen Staaten (Tschechoslowakei, Jugoslawien) verlief daher auch die Entwicklung von Ethnographie und Anthropologie völlig anders. Ihre Geschichtsschreibung ist in wesentlichen Bereichen oft noch zu erarbeiten, ${ }^{43}$ aber vorläufig kann festgehalten werden, dass hier die Phase nach Erlangung der staatlichen Selbstständigkeit fast durchwegs gleich zu setzen ist mit der Blütezeit einer nationalen Volkskunde. Von Irland über Norwegen und Finnland, von den baltischen Ländern über Polen bis zur

\footnotetext{
${ }^{40}$ BunzL, Matti: Franz Boas and the Humboldtian Tradition: From Volksgeist and Nationalcharakter to an Anthropological Concept of Culture, in: Stocking, George W. (ed.): Volksgeist as Method and Ethic: Essays on Boasian Ethnography and the German Anthropological Tradition, Madison: University of Wisconsin Press 1996, 17-78; Silvermann, Sydel: in: Barth et al.: One Discipline, Four Ways 2005, 261-273.

${ }^{41}$ Kuper, Adam: Anthropology and Anthropologists: The Modern British School, 3rd edition. London: Routledge 1996; Parkin, in: Barth et al.: One Discipline, Four Ways, 2005, 170-187.

42 KuKlick, Henrika (ed.): A New History of Anthropology, Oxford: Blackwell 2008, passim.

${ }^{43}$ Boskovic, Aleksandar (ed.): Other people's anthropologies: Ethnographic practice on the Margins, Oxford: Berghahn 2008.
} 
Tschechoslowakei, und von Ungarn über den Balkan bis in die Türkei ist für die 1920er- und 1930er-Jahre zu konstatieren: Verständlicherweise lag hier das absolute Primat auf der Dokumentation und Ausstellung der eigenen Nationalkulturen. Demgegenüber war die physische Anthropologie schon aufgrund der schwächer ausgebildeten eigenen naturwissenschaftlichen Kapazitäten meist eher nachrangig (mit einzelnen wichtigen Ausnahmen wie etwa Ungarn), während die Kulturen außerhalb des eigenen nationalen Bereiches kaum Gegenstand der Forschung waren. Einzelne Forschungsreisende bildeten ebenso markante Ausnahmen wie manche post-imperialen Bestände, etwa in den Völkerkunde-Sammlungen von Helsinki, Prag und Budapest. Eine führende Rolle der heimischen Volkskunde, eine nachgeordnete, teilweise damit verbundene Rolle der physischen Anthropologie, und seltene Lokalformen von Völkerkunde bildeten also das nord-, ost- und südosteuropäische Grundmuster der ethnographisch-anthropologischen Disziplinenlandschaft nach dem Ersten Weltkrieg. ${ }^{44}$ Davon wiederum deutlich abgehoben war jener Weg, der in der Sowjetunion nach 1917 beschritten wurde, und auf dem eine marxistisch-evolutionistische Ethnographie Vorrang hatte gegenüber einer zur Bedeutungslosigkeit degradierten physischen Anthropologie. ${ }^{45}$

Dass sich im deutschsprachigen Raum nach 1918 also ein nationaler und quasi-nationaler Sonderweg für Anthropologie und Ethnographie herausbildete, kann keinesfalls als auffällige Besonderheit angesprochen werden. Vielmehr war dies für den betreffenden Fachbereich, und keineswegs nur für diesen, eine völlig übliche Entwicklung des angebrochenen „kurzen“ 20. Jh. Ebenso wenig erstaunlich ist es, dass dieser deutschsprachige Sonderweg teils solche Elemente enthielt, welche eher bei den westlichen Großmächten üblich waren, teils aber auch solche, die eher typisch waren für die im Norden, Osten und Südosten entstandene neue politische Landschaft von selbstständigen Staaten. Ein guter Teil des deutschsprachigen Sonderwegs in Ethnographie und Anthropologie nach 1918 lag also völlig im Bereich des normalen Zeitgeistes damaliger Wissenschaftsentwicklungen Europas und Nordamerikas. Dieser erste Befund des vorliegenden Textes verdient ausdrücklich unterstrichen zu werden - auch, um mehr oder minder dämonisierenden Tendenzen einer teleologischen Historiographie in der Fachgeschichte entgegen zu wirken, wie sie in letzter Zeit da oder dort aufgetreten sind. ${ }^{46}$

Die institutionelle und disziplinäre Gliederung des früheren Fächerbereichs, Ethnographie und Anthropologie“ (bis 1914/18) stellte sich um 1928 im deutschsprachigen Raum als neue Dreigliederung mit stark veränderter Gewichtung dar. Die alte „Ethnographie“ war als Fachname endgültig verschwunden; als Bezeichnung wirkt sie seither auch auf Deutsch nur mehr fort für die spezifische Methode der ethnographischen Feldforschung. Aus der Ethnographie entstanden waren die zwei ungleich großen Fächer Volkskunde und Völkerkunde, mit ihren Museen und vor allem mit ihren neuen Universitätsinstituten. Diese Entwicklung, die in Deutschland bereits vor 1914 deutlicher hervorgetreten war, konnte sich nun nach der Auflösung der Monarchien und nach dem Verlust der Kolonien endgültig etablieren. Dabei repräsentierte die Errichtung einer selbstständigen Volkskunde gleichsam das (nord-, südost- und) „osteuropäische“ Element dieser Entwicklung innerhalb des deutschsprachigen Raumes, in dem ja nun ebenfalls, wenn auch unfreiwillig, so doch „neue Staaten ohne Kolonien" existierten. Dementsprechend stärker vertreten war die Volkskunde in Deutschland und Österreich ab 1918 auf der Ebene der regionalen Museen, aber allmählich auch auf der Ebene der Universitätsinstitute. ${ }^{47}$

Die Völkerkunde benötigte etwas länger, um sich universitär zu etablieren - um 1928 waren aber immerhin bereits vier solcher Institute eingerichtet (Berlin, Frankfurt, Leipzig, Wien). In institutioneller Hinsicht kann dies gleichsam als das „,westeuropäische“ Element in der betreffenden akademischen Landschaft angesprochen werden: Es entsprach nicht nur der eigenen imperialen und kolonialen Geschichte und ihrem Erbe, sondern auch dem Selbstverständnis von kulturellen Metropolen, dass diese ähnlich wie Frankreich, Großbritannien und die USA eigene Museen und eigene universitäre Fächer aufweisen, die sich mit außereuropäischen

\footnotetext{
${ }^{44}$ Gingrich, Andre: After the Great War, in Johler et al. 2010, 363-366.

${ }_{45}$ Moligner, Marina: Russian Physical Anthropology in Search of ,Imperial Race $:$ Liberalism and Modern Scientific Imagination in the Imperial Situation, in: Ab Imperio 8/1 2007, 191-223; SchweItzer, Peter: Siberia and Anthropology: National Traditions and Transnational Moments in the History of Research, Habil.schrift Univ. Wien 2001.

${ }^{46}$ Zimmermann, Andrew: Anthropology and Antihumanism 2001.

${ }^{47}$ JohLeR, Reinhard: Laboratory Conditions: German-speaking Volkskunde and the Great War, in Johler et al.: Anthropology in Wartime 2010, 136-140.
} 
Kulturen auseinander setzen. Inhaltlich etablierten sich dabei in Deutschland und Österreich zunehmend jene Versionen von Kulturkreislehre, deren Formierung bereits im Jahrzehnt vor dem Ersten Weltkrieg ihren Ausgang genommen hatte. ${ }^{48}$

Die eigentlich schwierige Frage des deutschsprachigen Sonderwegs in Ethnographie und Anthropologie nach 1918 ist also nicht die - aus heutiger Sicht längst nicht mehr zeitgemäße - Aufspaltung von Ethnographie in Volks- und Völkerkunde, und sie besteht auch nicht in der disziplinären Dreiteilung an sich. Vielmehr ist sie in der absolut dominanten Rolle der physischen Anthropologie innerhalb dieser Dreiteilung zu sehen. Diese hatte keinerlei Pendant im Westen oder im Osten, sie entsprach auch nicht dem inneren Erkenntnisstand von Volks- und Völkerkunde selbst. Gerade in Wien hatte die von Pater Wilhelm Schmidt forcierte, theologische Spielart der Kulturkreislehre als „Wiener Schule der Völkerkunde“ auch aus weltanschaulichen Gründen tiefe Vorbehalte gegenüber jeglichem einseitigen Primat der physischen Anthropologie. ${ }^{49}$

In den seit längerem etablierten wissenschaftlichen Gesellschaften und ebenso in zahlreichen neuen akademischen Organisationen der Zeit, in den Geldflüssen für die Forschung und in den Initiativen zur Gründung neuer Forschungsinstitutionen Deutschlands und Österreichs ist allerdings bereits in den späten 1920er Jahren ein anhaltendes Übergewicht der physischen Anthropologie deutlich konstatierbar, worauf hier nur über die betreffende Sekundärliteratur verwiesen werden kann. ${ }^{50}$ Markantester Höhepunkt dafür ist in den späten 1920er-Jahren die Gründung des Kaiser Wilhelm-Instituts für Anthropologie, menschliche Erblehre und Eugenik 1927 unter Leitung von Eugen Fischer. ${ }^{51}$ Neben einer Reihe von außerwissenschaftlichen Faktoren hat dieser Beitrag auch zwei wissenschaftsinterne und historisch aus dem Ersten Weltkrieg heraus gewachsene Faktoren identifiziert, die diesen ,hausgemachten“ Aspekt des Sonderwegs von Ethnographie und Anthropologie nach 1918 im deutschsprachigen Raum mit bedingt hatten.

Erstens war die Dominanz der physischen Anthropologie gegenüber Volks- und Völkerkunde Ende der 1920er Jahre kein Novum, sondern ein unmittelbares und praktisches Ergebnis der singulären Transformationen des Faches im und durch den Ersten Weltkrieg. Zweitens hatten viele jener Fachvertreter, die zu Ende der 1920er Jahre führende Stellungen in ihren akademischen Bereichen einnahmen, als junge Wissenschafter aktiv an den Kriegsgefangenen-Forschungen mitgewirkt und waren durch sie geformt worden.

\section{ZUSAMMENFASSUNG}

Der Erste Weltkrieg bedingte in vielen kultur- und geisteswissenschaftlichen akademischen Disziplinen aller Seiten eine verstärkte Auseinander-Entwicklung in Form von nationalen und quasi-nationalen theoretischen und methodischen Ausrichtungen der Fächer in den Institutionen jener Länder, die nun gegeneinander Krieg führten. Was zuvor noch als normale wissenschaftliche Konkurrenz in spätimperialen Zeiten funktioniert hatte, schottete sich nun zusehends voneinander ab. Im Bereich der deutschsprachigen Ethnographie und Anthropologie, aber auch im selben Fachbereich einiger Verbündeter, wurde diese Abschottung ergänzt durch präzedenzloses und - im Unterschied zu den Kriegsgegnern - singuläres Engagement im Krieg, und mithilfe des Krieges. Herausragend waren in dieser Hinsicht die groß angelegten Forschungen von Anthropologen und Ethnographen in den Kriegsgefangenen-Lagern des Ersten Weltkriegs.

\footnotetext{
${ }^{48}$ Gingrich, Andre and Steger, Brigitte: Kulturkreis 2011.

49 Marchand, Susan: Priest among the Pygmies: Wilhelm Schmidt and the Counter-Reformation in Austrian Ethnology, in: Penny and BunzL (eds.): Worldly Provincialism, 2003, 283-316.

${ }^{50}$ BERNER, Margit: From „Prisoners of War Studies“ to Proof of Paternity: Racial Anthropologists and the Measuring of ,others“ in Austria, in: TuRdA, M. and Weinduing, P. (eds.): 'Blood and Homeland': Eugenics and Racial Nationalism in Central and Southeast Europe 19001940, Budapest: Central European University Press 2006, 41-54; Evans, Andrew D.: World War I and the Science of Race in Germany, 2010; Proctor, Robert: From Anthropologie to Rassenkunde in the German Anthropological Tradition, in: Stocking, George W. (ed.): Bones, Bodies, Behavior: Essays on Biological Anthropology. Madison: University of Wisconsin Press 1988, 138-179.

${ }^{51}$ Siehe dazu Lösch, Niels: Rasse als Konstrukt 1997; MASsIN, Benoit: Rasse und Vererbung als Beruf. Die Hauptforschungsrichtungen am Kaiser-Wilhelm-Institut für Anthropologie, menschliche Erblehre und Eugenik im Nationalsozialismus, in: Schmuhl, HansWalter (Hg.): Rassenforschung an Kaiser-Wilhelms-Instituten vor und nach 1933, Göttingen: Wallstein 2003, 190-243.
} 
Die „Krise“ des Ersten Weltkrieges führte in Deutschland und Österreich über den Weg der methodischen und organisatorischen Implementierung dieser Kriegsgefangenen-Studien zu einer speziellen Art der „Transformation“ der Fächer Anthropologie und Ethnographie. Damit traten die deutschsprachigen Versionen dieser Fächer ein in eine Periode, die in weiten Bereichen der weltweiten Geistes-, Sozial- und Kulturwissenschaften schlechthin von nationalen Sonderformen geprägt war. Das Bemerkenswerte an der deutschsprachigen Transformation von Ethnographie und Anthropologie lag also weniger daran, dass auch sie in eine quasi-nationale (eigentlich: sprachräumliche) Sonderform mündete, und dass dies hier in Gestalt einer neuen disziplinären Dreiteilung erfolgte. Diese deutschsprachige Sonderform spiegelte zum Teil westliche, und zum Teil auch neue nord- und osteuropäische Entwicklungen wieder.

Vielmehr lag die Auffälligkeit dieses Sonderwegs in der radikalen Dominanz, die innerhalb der neuen Dreiteilung von der physischen Anthropologie gegenüber Volkskunde und Völkerkunde beansprucht und etwa Ende der 1920er Jahre dann auch personell, finanziell, institutionell und inhaltlich eingenommen wurde. Diese disziplinäre Vormachtstellung konnte die physische Anthropologie zur selben Zeit in der akademischen Welt nirgendwo sonst gegenüber den ethnographischen Fächern erringen. Neben einer Reihe von gesellschaftlichen und ideologischen Faktoren, welche diesen Aufstieg der deutschsprachigen physischen Anthropologie bedingten, lassen sich zwei ursächliche Faktoren dafür auch im innerwissenschaftlichen Bereich anhand der fachlichen Transformation durch den Ersten Weltkrieg identifizieren. Die Kriegsgefangenen-Lagerforschungen waren eine, wenn nicht überhaupt die große empirische Schulung einer ganzen Generation junger Ethnographen und Anthropologen, deren wissenschaftliche Laufbahnen nach 1918 nachhaltig davon geprägt blieben. Sie waren noch in beiden Fächern ausgebildet worden, das heißt sowohl als Kulturwissenschafter (Ethnographen) als auch als Lebensund Naturwissenschafter (Anthropologen). Allerdings mussten sie während und durch die Lagerforschungen die Erfahrung machen, dass die empirische Umsetzung der anthropologischen Aufgabenstellung rasch und sicher bewerkstelligt werden konnte, während ihnen für die Umsetzung der ethnographischen Aufgabenstellungen in den Lagern nahezu alle Voraussetzungen fehlten. Im Ergebnis resultierte daraus eine rasante Bevorzugung der Anthropologie, zuungunsten der aufwendigeren und schwierigeren Ethnographie - die zudem ein Minimum an Partizipation durch die beforschten Personen erfordert, während in den Lagern geradezu kontraproduktive Umstände dafür gegeben waren. Die auf Zwang, Mangel, Anonymität und Hierarchie beruhenden Umstände der Kriegsgefangenen-Lager selbst förderten damit eine einseitige Bevorzugung der physischen Anthropologie gegenüber der Partizipations-orientierten Ethnographie. Damit war zugleich eine Generation von JungWissenschaftern formativ geprägt, die ihre Karriere mit diesem bleibenden Vorverständnis antraten.

Abschließend stellt sich die Frage, inwiefern die eindeutige Transformation von Ethnographie und Anthropologie durch Krise und Fanal des Ersten Weltkrieges zugleich auch Elemente von Innovation enthielt. Das inkludiert zugleich auch die Frage nach dem Bedeutungsgehalt des Begriffs „Innovation“ für den Gang der Wissenschaften. Da dieser Begriff in Wissenschaftspolitik, Forschungsförderung, aber auch in der Werbewirtschaft der Gegenwart auf höchst unterschiedliche und nicht immer sinnvolle Weise zur Verwendung kommt, verspricht eine wissenschaftshistorische Sicht der Dinge hier einige Klärungen. Würde man nämlich unter „Innovation“ jegliche ,neue“ Entwicklung in den Wissenschaften verstehen, die eine mehr oder minder grundsätzliche Abkehr vom bis dahin Vorherrschenden anzeigt, dann wäre von „Innovation im weitestmöglichen Sinn“"zu sprechen. In diesem weit definierten Verständnis von Innovation wäre dann jegliche grundsätzlichere Transformation im Wissenschaftsbetrieb zugleich synonym mit Innovation zu setzen.

Daraus ergäbe sich nicht nur das terminologische Problem mangelnder Trennschärfe zwischen den zwei Begriffen „Transformation“ und „Innovation“, sondern auch ein sachliches und faktisches Problem. Es kann wohl wenig Sinn machen, eine damals ,neuartige“ Entwicklung in den Wissenschaften als „Innovation“ zu bezeichnen, wenn aus heutiger Sicht zugleich klar ist, dass sie in eine Sackgasse führte. Aus dieser Sicht der Gegenwart nämlich brachte das Primat der physischen Anthropologie über Volks- und Völkerkunde keinerlei „Weiterentwicklung“ innerhalb dieser Wissenschaften. Ebenso ist es aus heutiger Sicht kaum als positive Weiterentwicklung nach 1918 zu bezeichnen, dass Volks- und Völkerkunde sich als voneinander unabhängige akademische Fächer etablieren konnten. Vielmehr sollte beides, die Trennung der Volks- und Völkerkunde voneinander und die Unterordnung der beiden getrennten Fächer unter die physische Anthropologie, sich als wichtige - und so noch nicht a priori beabsichtigte - innerakademische 
Weichenstellung bei einer weiter gehenden Entwicklung erweisen, die letztlich zur Machtübernahme der NS-Ideologie im Wissenschaftsbereich führen sollte.

Neben diesen institutionellen Dimensionen der Transformation ist auch auf ihre inhaltlichen Komponenten zu verweisen. Vieles von dem, was Volks- und Völkerkunde während und nach dem Ersten Weltkrieg erarbeiteten, kann aus heutiger Sicht noch mit einigem Gewinn und bei entsprechender Quellenkritik konsultiert werden. Das gleiche lässt sich für die physische Anthropologie kaum behaupten. Insofern bietet uns die physische Anthropologie 1914-18 auch das Beispiel eines Vorläufers zu manchen aktuellen Entwicklungen der Gegenwart. Die großen Ankündigungen der physischen Anthropologie erwiesen sich nämlich schon kurzund mittelfristig fast durchwegs als leere Versprechungen, und auch langfristig sollte sich der kumulative und epistemologische Wert der Vermessungen in den Kriegsgefangenen-Lagern von 1914-18 auf Null belaufen. Dieser wissenschaftshistorische Befund ist geeignet, unsere Kritikfähigkeit heute auch dort zu artikulieren, wo Natur- und Lebenswissenschaften ihre verständlichen Wünsche nach Mittelerhöhungen glauben, auf Kosten der Kultur- und Geisteswissenschaften vortragen zu müssen.

\section{LITERATURVERZEICHNIS}

Aснам, Karl (Hg.): Geschichte der österreichischen Humanwissenschaften, Bd. 4: Geschichte und fremde Kulturen. Wien: Passagen 2002, 323-368.

Ballhausen, Thomas, Matiasek, Katarina, Teschler-Nicola, Maria (Hg.): Archivhorizonte: Wissenslandschaften und Perspektivgrenzen im multimedialen Nachlass des Anthropologen und Forschungsreisenden Rudolf Pöch, Wien: Löcker i.Dr. Gingrich in Barth et al.: One Discipline, Four Ways 2005, 74.

Barth, Fredrik, Gingrich, Andre, Parkin, Robert, Silvermann, Sydel: One Discipline, Four Ways: British, German, French, and American Anthropology, Chicago: Chicago University Press 2005.

BAuer, Karl J.: Alois Musil: Wahrheitssucher in der Wüste, Wien: Böhlau 1989.

BENDIX, Regina: Ethnology, Cultural Reification, and the Dynamics of Difference in the Kronprinzenwerk.

BERNER, Margit: Large-Scale Anthropological Surveys in Austria-Hungary, 1871-1918, in Johler et al.: Anthropology in Wartime 2010, $233-254$.

Berner, Margit: From „Prisoners of War Studies“ to Proof of Paternity: Racial Anthropologists and the Measuring of „others“ in Austria, in: Turda, M. and Weindling, P. (eds.): 'Blood and Homeland': Eugenics and Racial Nationalism in Central and Southeast Europe 1900-1940, Budapest: Central European University Press 2006, 41-54.

Boskovic, Aleksandar (ed.): Other people's anthropologies: Ethnographic practice on the Margins, Oxford: Berghahn 2008.

BRAUKÄMPER, Ulrich: Im Spannungsfeld zwischen Wissenschaft und politischem Aktivismus: Leo Frobenius als Geheimagent in Nordost-Afrika, in: Kohl, Karl-Heinz und Platte, Editha (Hg.): Gestalter und Gestalten: 100 Jahre Ethnologie in Frankfurt am Main, Frankfurt - Basel: Stroemfeld, 167-186.

BunzL, Matti: Franz Boas and the Humboldtian Tradition: From Volksgeist and Nationalcharakter to an Anthropological Concept of Culture, in: Stocking, George W. (ed.): Volksgeist as Method and Ethic: Essays on Boasian Ethnography and the German Anthropological Tradition, Madison: University of Wisconsin Press 1996, 17-78.

Cole, Douglas: Franz Boas: The early years. 1858-1906. University of Washington Press: London - Seattle 1999.

Evans, Andrew D.: Science behind the Lines: The Effects of World War I on Anthropology in Germany, in: Johler et al.: Anthropology in Wartime 2010, 115-116

Evans, Andrew D.: World War I and the Science of Race in Germany. Chicago: Chicago University Press 2010.

Evans, Andrew: Science behind the Lines, in Johler et al.: Anthropology in Wartime 2010, 115; BerNer, Margit: Large-Scale Anthropological Surveys in Austria-Hungary, 1871-1918, in: Johler et al.: Anthropology in Wartime 2010, $248-252$.

FEIGL, Erich: Musil von Arabien: Vorkämpfer der islamischen Welt, Frankfurt/M.: Ullstein 1988.

Gellner, Ernest: Anthropology and Politics: Revolutions in the Sacred Grove, Oxford: Blackwell-Wiley 1995, 212-228; Gessler, Bernhard: Eugen Fischer (1874-1967). Leben und Werk des Freiburger Anatomen, Anthropologen und Rassenhygienikers bis 1927. Frankfurt/Main: Peter Lang Verlag 2000.

Geisenhainer, Katja: „Rasse ist Schicksal.“ Otto Reche (1879-1966) - ein Leben als Anthropologe und Völkerkundler. Beiträge zur Leipziger Universitäts- und Wissenschaftsgeschichte, Reihe A Band 1, Leipzig: Evangelische Verlagsanstalt 2002; JANATA, Alfred: Jemen - Im Land der Königin von Saba. Wien: Museum für Völkerkunde 1989.

Gingrich, Andre: After the Great War: National Re-Configurations of Anthropology in Late Colonial Times, in: Johler, Reinhard, Marchetti, Christian, Scheer, Monique (eds.): Doing Anthropology in Wartime and War Zones, Bielefeld: transcript $2010,355-379$.

Gingrich, Andre und Lange, Britta: Gefangene Stimmen, Internierte Körper: Rudolf Pöch, die Wünsdorf-Reise 1917.

Gingrich, Andre und Steger, Brigitte: Kulturkreis, in: Kreff, Fernand, Knoll, Eva-Maria, Gingrich, Andre (Hg.): Lexikon zur Globalisierung: Anthropologische und sozialwissenschaftliche Zugänge zur Praxis, Bielefeld: transcript 2011, pp. $217-219$.

GINGRICH, Andre: Liberalism in imperial anthropology: Notes on an implicit paradigm in continental European anthropology before World War I, in: Ab Imperio 1/2007, 224-239.

Gingrich, Andre and STEgER, Brigitte: Kulturkreis 2011. 
GirTLER, Roland: Franz Boas, Burschenschaftler und Schwiegersohn eines österreichischen Revolutionärs von 1848, Anthropos 96/2001, 572.

Johler, Reinhard, Marchetti, Christian, Scheer, Monique (eds.): Doing Anthropology in Wartime and War Zones, Bielefeld: transcript 2010, 355-379.

JoHLER, Reinhard: Laboratory Conditions: German-speaking Volkskunde and the Great War, in Johler et al.: Anthropology in Wartime 2010, 136-140.

JuNG, Peter: Der k.u.k. Wüstenkrieg: Österreich-Ungarn im Vorderen Orient, 1915-1918, Graz: Styria: Militärhistor. Dienst, 1992.

Koepping, Klaus Peter: Adolf Bastian and the Psychic Unity of Mankind: The Foundations of Anthropology in Nineteenth Century Germany. St. Lucia: University of Queensland Press 1983.

Kuklick, Henrika (Hg.): A New History of Anthropology, Oxford: Blackwell 2008.

KUPER, Adam: Anthropology and Anthropologists: The Modern British School, 3rd edition. London: Routledge 1996; Parkin, in: Barth et al.: One Discipline, Four Ways, 2005, 170-187.

LANGE, Britta: AfterMath: Anthropological Data from Prisoner-of-War Camps, in Johler et al.: Anthropology in Wartime 2010, 311-335.

LANGE, Britta und Gingrich, Andre: Wünsdorf-Reise 1917, in: Ballhausen et al. (i.Dr.).

LoEsCH Niels C.: Rasse als Konstrukt. Leben und Werk Eugen Fischers. Frankfurt/Main: Peter Lang Verlag 1997.

Manias, Chris: The Race Prussienne Controversy: Scientific Internationalism and the Nation, in: Isis 100 (2009), 733-757.

Marchand, Susan: Priest among the Pygmies: Wilhelm Schmidt and the Counter-Reformation in Austrian Ethnology, in: Penny and BunzL (eds.): Worldly Provincialism, 2003, 283-316.

Marchetti, Christian: Austro-Hungarian Volkskunde at War: Scientists on Ethnographic Mission in World War I, in Johler et al.: Anthropology in Wartime 2010, 207-230.

Massin, Benoit: From Virchow to Fischer: Physical anthropology and „modern race theories“ in Wilhelmine Germany, in: STOCKING, George W. Jr. (ed.): Volksgeist as Method and Ethic: Essays on Boasian ethnography and the German anthropological tradition, History of Anthropology vol. 8, Madison: University of Wisconsin Press 1996, 79-154.

MAsSIN, Benoit: Rasse und Vererbung als Beruf. Die Hauptforschungsrichtungen am Kaiser-Wilhelm-Institut für Anthropologie, menschliche Erblehre und Eugenik im Nationalsozialismus, in: Schmuhl, Hans-Walter (Hg.): Rassenforschung an Kaiser-WilhelmsInstituten vor und nach 1933, Göttingen: Wallstein 2003, 190-243.

Moligner, Marina: Russian Physical Anthropology in Search of ,Imperial Race': Liberalism and Modern Scientific Imagination in the Imperial Situation, in: Ab Imperio 8/1 2007, 191-223.

MueHLFRIED, Florian: R. Bleichsteiners ,Kaukasische Forschungen', in Georgica 2002/24, 51-58.

PARKIN in Barth, Fredrik et al. 2005, 170-185.

Penny, Glenn H. and Bunzl, Matti (eds.): Worldly Provincialism: German anthropology in the age of empire. Ann Arbor: University of Michigan Press 2003.

Price, David H.: Anthropological Intelligence. The Deployment and Neglect of American Anthropology in the Second World War, Durham - London: Duke University Press 2008.

Proctor, Robert: From Anthropologie to Rassenkunde in the German Anthropological Tradition, in: Stocking, George W. (ed.): Bones, Bodies, Behavior: Essays on Biological Anthropology. Madison: University of Wisconsin Press 1988, 138-179.

Pusman, Karl: Die "Wissenschaften vom Menschen" auf Wiener Boden (1870-1959): Die Anthropologische Gesellschaft in Wien und die anthropologischen Disziplinen im Fokus von Wissenschaftsgeschichte, Wissenschafts- und Verdrängungspolitik, Münster: LiT 2008.

Rauchensteiner, Manfried: Der Tod des Doppeladlers: Österreich-Ungarn und der Erste Weltkrieg, 2. Auflage Graz: Steirische Verlagsgesellschaft 1998.

RugGendorfer, Peter und Szemethy, Hubert D.: Felix von Luschan (1854-1924): Leben und Wirken eines Universalgelehrten, Wien: Böhlau 2009; zu Thurnwald siehe: Mецк-Косн Marion: Auf der Suche nach der menschlichen Gesellschaft: Richard Thurnwald, Berlin: Reimer 1989; zu Pöch siehe Ballhausen et al.: Archivhorizonte: Wien: Löcker i.Dr.

SCHEER, Monique: Captive Voices: Phonographic Recordings in the German and Austrian Prisoner-of-War Camps of World War I, in: Johler et al.: Anthropology in Wartime 2010, 287-308.

SchweItzer, Peter: Siberia and Anthropology: National Traditions and Transnational Moments in the History of Research, Habil.schrift Univ. Wien 2001.

Stachel, Peter: Die Harmonisierung national-politischer Gegensätze und die Anfänge der Ethnographie in Österreich.

Silvermann, Sydel: in: Barth et al.: One Discipline, Four Ways 2005, 261-273.

Six-Hohenbalken, Maria: "A moral witness within complicity" - Edmund Jaroljmeks Tagebuchaufzeichnungen aus Mosul 1916. Ein Beitrag zur Anthropologie von Gewalt, in: Anthropos 107/2012: 71-86.

TuRDA, Marius: The Idea of National Superiority in Central Europe, 1880-1918. New York: Edwin Mellen Press 2005.

Vermeulen, Han: Origins and Institutionalization of Ethnography and Ethnology in Europe and the USA, 1771-1845.

Vermeulen, Han and Roldan, Arturo Alvarez (eds.): Fieldwork and Footnotes: Studies in the History of European Anthropology, London: Routledge 1995, 39-59.

Wingfield, Nancy M. (ed.): Creating the Other: Ethnic Conflict and Nationalism in the Habsburg Empire. New York - London: Berghahn $2003,149-165$.

Young, Michael W.: Malinowski: Odyssey of an Anthropologist, 1884-1920, New Haven: Yale UP 2004.

Zамміто, John H.: Kant, Herder and the Birth of Anthropology, Chicago: University of Chicago Press 2002.

Zimmermann, Andrew: Anthropology and Antihumanism in Imperial Germany. Chicago - London: University of Chicago Press 2001.

Zitelmann, Thomas: Des Teufels Lustgarten: Themen und Tabus der politischen Anthropologie Nordostafrikas. Habil.schrift FU Berlin 1999. 
